Louisiana State University

LSU Digital Commons

Faculty Publications

Department of Physics \& Astronomy

6-1-2004

\title{
Interstellar polarization in M31
}

\author{
Geoffrey C. Clayton \\ Louisiana State University \\ Michael J. Wolff \\ Space Science Institute \\ Karl D. Gordon \\ The University of Arizona \\ Paul S. Smith \\ The University of Arizona \\ Kenneth H. Nordsieck \\ University of Wisconsin-Madison
}

See next page for additional authors

Follow this and additional works at: https://digitalcommons.Isu.edu/physics_astronomy_pubs

\section{Recommended Citation}

Clayton, G., Wolff, M., Gordon, K., Smith, P., Nordsieck, K., \& Babler, B. (2004). Interstellar polarization in M31. Astronomical Journal, 127 (6 1782), 3382-3387. https://doi.org/10.1086/420714

This Article is brought to you for free and open access by the Department of Physics \& Astronomy at LSU Digital Commons. It has been accepted for inclusion in Faculty Publications by an authorized administrator of LSU Digital Commons. For more information, please contact ir@lsu.edu. 
Authors

Geoffrey C. Clayton, Michael J. Wolff, Karl D. Gordon, Paul S. Smith, Kenneth H. Nordsieck, and Brian L. Babler 


\author{
INTERSTELLAR POLARIZATION IN M31 ${ }^{1}$ \\ Geoffrey C. Clayton, ${ }^{2}$ Michael J. Wolff, ${ }^{3}$ Karl D. Gordon, ${ }^{4}$ Paul S. Smith, ${ }^{4}$ \\ Kenneth H. Nordsieck, ${ }^{5}$ and Brian L. BableR ${ }^{5}$ \\ Received 2004 January 5; accepted 2004 February 23
}

\begin{abstract}
The wavelength dependence of interstellar polarization due to dust in M31 has been observed along four sight lines. Only one sight line had been measured previously. The globular clusters, S78, S150, S233, and Baade 327 were used as point sources to probe the interstellar dust in M31. The Serkowski law produces good fits for all the sight lines, although the relationship between $K$ and $\lambda_{\max }$ may be different from that found in the Galaxy. The results of this study imply that the slope $K / \lambda_{\max }$ may be significantly larger in M31. The Serkowski curves are significantly narrower than those of the same $\lambda_{\max }$ in the Galaxy and may require extreme modifications to the size distributions of silicate particles. The fits for the four sight lines reveal values of $\lambda_{\max }$ ranging from 4800 to $5500 \AA$. These are consistent with average values of $\lambda_{\max }$ measured in the Galaxy and the Magellanic Clouds. The range measured for M31 corresponds to $R_{V}$ values of 2.7-3.1. The range in $R_{V}$ seen in the Galaxy is 2.5-5.5 implying, for this small sample, that the average size of interstellar grains in M31 is typically smaller than that seen for Galactic grains if the nature of the grains is the same. Also, the polarization efficiency for these sight lines is large, although some bias is expected since sight lines known to have significant interstellar polarization were selected for the sample.
\end{abstract}

Key words: dust, extinction — galaxies: individual (M31) — polarization

\section{INTRODUCTION}

There is an average Milky Way extinction relation, depending on only one parameter, the ratio of total-to-selective extinction, $R_{V}$, which is applicable to a wide range of interstellar dust environments (Cardelli, Clayton, \& Mathis 1989, hereafter CCM). Similarly, there is an interstellar polarization relation, correlated with a single parameter, $\lambda_{\max }$, the wavelength at which the interstellar polarization is a maximum (Serkowski, Mathewson, \& Ford 1975; Clayton et al. 1995; Martin, Clayton, \& Wolff 1999). The parameters, $R_{V}$ and $\lambda_{\max }$, both involve integrals over the size distribution of interstellar dust grains and are well correlated with each other (Serkowski et al. 1975; Whittet \& van Breda 1978; Clayton \& Mathis 1988). The existence of these relations, valid over a large wavelength interval, suggests that the environmental processes that modify the grains are efficient and affect all grains. However, the CCM relation is not a universal law. It does not fit the observed extinction in the sight lines observed in the Magellanic Clouds (e.g., Clayton et al. 1996; Gordon \& Clayton 1998; Misselt, Clayton, \& Gordon 1999; Gordon et al. 2003). The differences in observed extinction properties seen among the Local Group galaxies may be due to various environmental factors including metallicity. The metallicity of the LMC and SMC is lower than the Galaxy. Therefore, the nature of the dust in M31 is of great interest because its metallicity is similar to that in the Milky Way.

\footnotetext{
${ }^{1}$ A portion of the results presented here made use of the Multiple Mirror Telescope Observatory, a facility operated jointly by the University of Arizona and the Smithsonian Institution.

${ }^{2}$ Department of Physics and Astronomy, Louisiana State University, Baton Rouge, LA 70803; gclayton@fenway.phys.lsu.edu.

3 Space Science Institute, 1540 30th Street, Suite 23, Boulder, CO 803031012; wolff@colorado.edu.

${ }^{4}$ Steward Observatory, University of Arizona, 933 North Cherry Avenue, Tucson, AZ 85721; kgordon@as.arizona.edu, psmith@as.arizona.edu.

5 Space Astronomy laboratory, University of Wisconsin-Madison, 1150 University Avenue, Madison, WI 53706; khn@sal.wisc.edu, brian@sal.wisc.edu.
}

The nature of interstellar dust in M31 is summarized in Lequeux (2000). The UV extinction data for M31, which are based on only a few lightly reddened sight lines, show a CCM-like far-UV extinction and a weak $2175 \AA$ bump (Bianchi et al. 1996). Several studies have suggested that the extinction in M31 in the $U$-band differs from the Galaxy (Searle 1983; Iye \& Richter 1985; Massey et al. 1995). It has also been suggested that there is a systematic change in the $U$-band extinction with distance from the center of M31 (Iye \& Richter 1985; Freedman \& Madore 1990). Various estimates of $R_{V}$ ranging from 2.5 to 3.8 have been made using individual stars and clusters in M31 (Kron \& Mayall 1960; Sharov \& Lyutyj 1989; Nedialkov \& Veltchev 1999). Recently, Barmby et al. (2000) find that the $U$ - to $K$-band extinction curve in M31, derived from a large number of globular clusters, is consistent with CCM with $R_{V}=3.1$.

The interstellar polarization in the LMC and SMC shows a similar wavelength dependence to that seen in the Milky Way and can be well fit by the Serkowski relation (Clayton, Martin, \& Thompson 1983; Clayton et al. 1996; Rodrigues et al. 1997). Some interstellar polarization measurements in M31 have been made using globular clusters as point sources (Hiltner 1958; Martin \& Shawl 1979, 1982). However, the wavelength dependence of interstellar polarization has been measured along only one line of sight (Martin \& Shawl 1979). This polarization measurement is consistent with the Serkowski relationship and a small value of $\lambda_{\max }$. We report further observations of the wavelength dependence of interstellar polarization in M31.

\section{OBSERVATIONS}

Optical spectropolarimetry was obtained using the $6.5 \mathrm{~m}$ telescope of the Multiple Mirror Telescope (MMT) Observatory located on Mount Hopkins, Arizona. Observations were made of four globular clusters listed in Table 1 on 2003 October 30 UT. All of the observations made use of the CCD Spectropolarimeter (Schmidt, Stockman, \& Smith 1992), upgraded 
TABLE 1

Observed M31 Globular Clusters

\begin{tabular}{|c|c|c|c|c|c|c|}
\hline Cluster $^{\mathrm{a}}$ & $V^{\mathrm{b}}$ & $E(B-V)^{\mathrm{b}}$ & $\begin{array}{l}p_{\max }^{\mathrm{c}} \\
(\%)\end{array}$ & $\begin{array}{c}\lambda_{\max }{ }^{c} \\
(\AA)\end{array}$ & $K^{\mathrm{c}}$ & $\begin{array}{c}p_{\max } / E(B-V)^{\mathrm{d}} \\
\left(\% \mathrm{mag}^{-1}\right)\end{array}$ \\
\hline ................... & 14.26 & $0.36 \pm 0.04$ & $1.96 \pm 0.03$ & $4775 \pm 250$ & $1.32 \pm 0.28$ & $7.5 \pm 0.15$ \\
\hline S150 ......................... & 15.44 & $0.48 \pm 0.06$ & $3.19 \pm 0.05$ & $5200 \pm 225$ & $1.78 \pm 0.46$ & $8.4 \pm 0.16$ \\
\hline S233 …….............. & 15.38 & $0.23 \pm 0.05$ & $1.99 \pm 0.03$ & $5275 \pm 175$ & $2.38 \pm 0.49$ & $15.3 \pm 0.39$ \\
\hline Baade $327 \ldots \ldots \ldots$ & 16.71 & $1.38 \pm 0.02$ & $9.14 \pm 0.12$ & $5450 \pm 175$ & $2.22 \pm 0.35$ & $7.1 \pm 0.02$ \\
\hline
\end{tabular}

${ }^{a}$ From Vetesnik 1962 and Sargent et al. 1977.

b Total reddening as estimated by Barmby et al. 2000 .

${ }^{c}$ Based on MMT data only.

${ }^{\mathrm{d}}$ Galactic foreground reddening of $E(B-V)=0.1$ subtracted.

with a $1200 \times 800$ pixel, thinned, antireflection-coated, UVsensitized CCD, and an improved camera lens and half-wave plate. All observations used a 600 line $\mathrm{mm}^{-1}$ grating for high throughput and wide spectral coverage, $4100-8300 \AA$. The FWHM resolution with this grating and a slit width of $1^{\prime \prime}$ is $\sim 17 \AA$ ( 3 pixels). A polarimetric measurement sequence involves four separate exposures that sample 16 orientations of the semiachromatic half-wave plate and totalled $480-1440 \mathrm{~s}$ of integration for each of the four clusters through light cloud cover. Polarization position angles were referenced to the equatorial system using interstellar polarization standards (Schmidt, Elston, \& Lupie 1992). The spectral flux distribution is also obtained in the course of the observations and is calibrated relative to spectrophotometric standard stars selected from the IRAF database (Massey et al. 1988). The data have been binned and are plotted in Figure 1 along with the spectra of the globular clusters.

In addition, spectropolarimetric observations of S78 were obtained with the HPOL spectropolarimeter on the $3.5 \mathrm{~m}$ Wisconsin-Indiana-Yale-NOAO (WIYN) telescope on Kitt Peak. The spectropolarimeter is a modified Boller \& Chivens spectrograph with a rotating superachromatic wave plate after the slit (Nordsieck \& Harris 1996; Wolff, Nordsieck, \& Nook 1996). Spectra are obtained at eight wave plate angles on a Reticon $400 \times 1200 \mathrm{CCD}$. Two gratings are used, producing spectra and 3 pixel resolutions of 3200-6200 $\AA(9 \AA)$ and 6000-10500 $\AA$ (12 $)$, respectively. Two polarized star spectra and two polarized sky spectra are obtained simultaneously. The calibration accuracy in polarization is $\pm p / 500$ and in position angle is $\pm 1^{\circ}$. There is an uncertainty of $0.1 \%$ in the correction for instrumental polarization. This small uncertainty does not affect the results presented here. The WIYN spectropolarimetric data were obtained in 1996 December and 1998 October. The four nights of data on S78 have been combined and binned to increase the signal-to-noise ratio $(\mathrm{S} / \mathrm{N})$. They are plotted in Figure 1.

The polarization measured toward M31 must be corrected for a foreground component. These lines of sight pass through the disk and halo of the Galaxy, and then the halo and disk of M31. However, since M31 lies at a Galactic latitude of $-22^{\circ}$, the foreground extinction due to dust in the Galaxy is small. Several estimates have been made of the reddening foreground to M31. They all indicate that $E(B-V) \sim$ 0.08-0.11 (Bianchi et al. 1996 and references therein). Using the well-known Galactic relationship between reddening and interstellar polarization, the maximum foreground polarization is $\sim 0.9 \%$ (Serkowski et al. 1975). Martin \& Shawl (1979) estimated the actual foreground polarization by measuring a sample of Galactic stars lying in the direction of M31, as well as the nucleus of M31 itself. The foreground dust is patchy, so there is no way to know a priori what the foreground component is along any given sight line. So, Martin \& Shawl (1982) adopted a best-guess value of $p_{\max }=0.4 \%, \lambda_{\max }=5500$ $\AA$ with a position angle of $90^{\circ}$ by averaging several foreground sight lines.

The new observations reported here have been corrected for foreground polarization in the same manner as Martin \& Shawl (1982). A Galactic Serkowski curve, as described above, was assumed and the foreground polarization at each wavelength was calculated and subtracted. The foregroundcorrected polarizations are plotted in Figure 1. The observed and foreground-corrected values are listed in Tables 2-6. The new data are consistent with the Martin \& Shawl (1982) data for S78 in the overlapping wavelength range. Similarly, the three wavelength bins for S150 and one bin for S233 agree with our new data within $2 \sigma$. There is only one filterless observation of Baade 327 having low S/N. The Martin \& Shawl filter data are plotted for comparison in Figure 1.

\section{DISCUSSION}

The interstellar polarization due to dust in M31 was first measured by Hiltner (1958) using globular clusters as point sources. He made unfiltered observations of about 20 clusters finding several with significant amounts of polarization. Filter polarimetry of a similar number of clusters was obtained by Martin \& Shawl $(1979,1982)$. Only one sight line in their sample, toward S78, had enough wavelength coverage for a Serkowski fit to be made. That sight line showed $p_{\max }=$ $1.98 \pm 0.07 \%$, and $\lambda_{\max }=3500 \pm 300 \AA$ using the Wilking et al. (1980) formulation of the Serkowski relation.

Our new data for S78 and three additional clusters cover approximately the same wavelength range (4100-8300 $⿱$ ) as Martin \& Shawl but with many more data bins and smaller uncertainties. We performed nonlinear least-squares fits to the foreground-corrected data for all four clusters using the Serkowski law (Serkowski et al. 1975; Wilking et al. 1980; Wilking, Lebofsky, \& Rieke 1982; Whittet et al. 1992). We found that the $K=1.66 \lambda_{\max }$ relationship, which holds for Galactic interstellar polarization, does not produce good fits for the M31 sight lines (Whittet et al. 1992). However, the Serkowski law with $K$ as a free parameter does produce good fits for all of the sight lines. The three fit parameters are listed in Table 1. The relationship between $K$ and $\lambda_{\max }$ is shown in Figure 2. Although, we do not have enough sight lines to define the $K-\lambda_{\max }$ relationship for M31, Figure 2 shows that the slope may be steeper than in the Galaxy.

The $K-\lambda_{\max }$ relationship for the M31 globular clusters is very interesting in that it produces distinctly narrower "Serkowski 

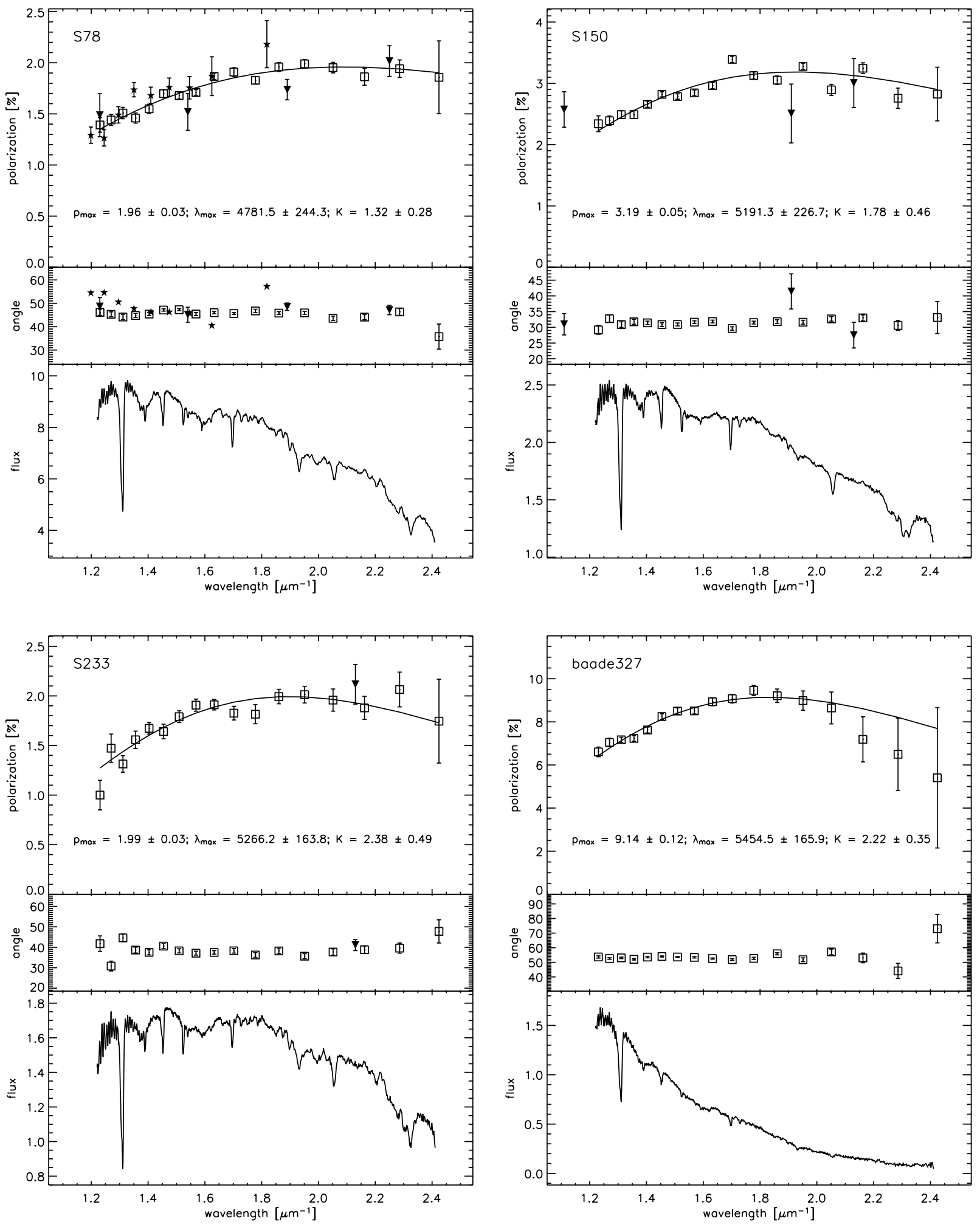

Fig. 1.-Spectropolarimetric observations of M31 globular clusters. Top, foreground corrected polarization; middle, position angle; bottom, the spectra. Open squares represent the MMT data, the stars represent the WIYN data, and the triangles represent the Martin \& Shawl (1982) data. The error bars are $1 \sigma$. The lines drawn represent the best Serkowski fits to the MMT data only. See text.

curves" than those of the same $\lambda_{\max }$ in the Galaxy. As has been shown many times in the past (Wolff et al. 1994; Clayton et al. 1995; Martin et al. 1999), grain models have generally been able to reproduce observed Galactic interstellar polarization curves by small changes to the size distribution of aligned grains, alignment efficiency, upper integration limit, etc. This is definitely not the case for the M31 curves in that similar changes to a distribution of astronomical silicates failed to produce $K$ values of the observed amplitude. Even more extreme modifications to the size distribution of silicate particles (use of "flat" distributions, very narrow size ranges, etc.) tended to produce $K$ values that still remained $30 \%-40 \%$ too 
TABLE 2

S78-MMT

\begin{tabular}{|c|c|c|c|c|}
\hline $\begin{array}{c}\lambda \text { bin } \\
(\AA)\end{array}$ & $\begin{array}{c}p \\
(\%)\end{array}$ & $\theta$ & $p_{\text {corr }}$ & $\theta_{\text {corr }}$ \\
\hline $4125 \ldots$ & $1.78 \pm 0.36$ & $41.5 \pm 5.4$ & 1.86 & 35.76 \\
\hline $4375 \ldots \ldots$ & $2.00 \pm 0.09$ & $51.8 \pm 1.5$ & 1.94 & 46.32 \\
\hline $4625 \ldots \ldots$ & $1.89 \pm 0.08$ & $50.0 \pm 1.1$ & 1.86 & 44.09 \\
\hline $4875 \ldots \ldots$ & $1.97 \pm 0.05$ & $49.3 \pm 0.9$ & 1.95 & 43.58 \\
\hline $5125 \ldots \ldots \ldots$ & $2.04 \pm 0.04$ & $51.5 \pm 0.6$ & 1.99 & 45.89 \\
\hline $5375 \ldots \ldots$ & $2.01 \pm 0.04$ & $51.5 \pm 0.6$ & 1.96 & 45.81 \\
\hline $5625 \ldots \ldots$ & $1.90 \pm 0.04$ & $52.8 \pm 0.7$ & 1.83 & 46.72 \\
\hline 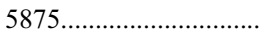 & $1.96 \pm 0.04$ & $51.5 \pm 0.4$ & 1.91 & 45.63 \\
\hline $6125 \ldots \ldots$ & $1.92 \pm 0.03$ & $51.9 \pm 0.5$ & 1.87 & 45.98 \\
\hline $6375 \ldots \ldots$ & $1.76 \pm 0.03$ & $51.8 \pm 0.7$ & 1.71 & 45.41 \\
\hline $6625 \ldots \ldots$ & $1.75 \pm 0.03$ & $53.6 \pm 0.4$ & 1.68 & 47.25 \\
\hline $6875 \ldots \ldots$ & $1.77 \pm 0.04$ & $53.3 \pm 0.5$ & 1.70 & 47.12 \\
\hline $7125 \ldots \ldots$ & $1.60 \pm 0.04$ & $52.2 \pm 0.6$ & 1.55 & 45.38 \\
\hline 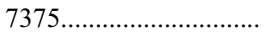 & $1.50 \pm 0.05$ & $51.9 \pm 0.8$ & 1.46 & 44.79 \\
\hline $7625 \ldots \ldots \ldots \ldots \ldots \ldots \ldots \ldots \ldots \ldots \ldots \ldots \ldots \ldots \ldots$ & $1.54 \pm 0.06$ & $50.9 \pm 1.0$ & 1.51 & 44.14 \\
\hline $7875 \ldots \ldots$ & $1.49 \pm 0.05$ & $52.2 \pm 1.0$ & 1.44 & 45.29 \\
\hline 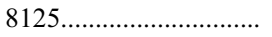 & $1.45 \pm 0.07$ & $53.0 \pm 1.4$ & 1.39 & 46.09 \\
\hline
\end{tabular}

TABLE 3

S78-WIYN

\begin{tabular}{|c|c|c|c|c|}
\hline $\begin{array}{c}\lambda \text { bin } \\
(\AA)\end{array}$ & $\begin{array}{c}p \\
(\%)\end{array}$ & $\theta$ & $p_{\text {corr }}$ & $\theta_{\text {corr }}$ \\
\hline ................. & $2.37 \pm 0.23$ & $61.7 \pm 2.8$ & 2.18 & 57.31 \\
\hline $6156 \ldots \ldots . .$. & $1.85 \pm 0.19$ & $46.7 \pm 2.9$ & 1.87 & 40.54 \\
\hline $6469 \ldots \ldots$ & $1.81 \pm 0.11$ & $52.1 \pm 1.7$ & 1.76 & 45.74 \\
\hline ................. & $1.82 \pm 0.09$ & $52.4 \pm 1.4$ & 1.76 & 46.18 \\
\hline $7094 \ldots .$. & $1.74 \pm 0.08$ & $52.5 \pm 1.3$ & 1.68 & 46.09 \\
\hline $7406 \ldots \ldots$ & $1.81 \pm 0.07$ & $53.6 \pm 1.1$ & 1.74 & 47.58 \\
\hline 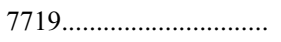 & $1.60 \pm 0.08$ & $57.0 \pm 1.4$ & 1.49 & 50.34 \\
\hline ................. & $1.42 \pm 0.08$ & $61.4 \pm 1.6$ & 1.26 & 54.30 \\
\hline 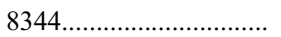 & $1.44 \pm 0.08$ & $61.0 \pm 1.6$ & 1.29 & 54.16 \\
\hline
\end{tabular}

TABLE 4

S150

\begin{tabular}{|c|c|c|c|c|}
\hline $\begin{array}{c}\lambda \text { bin } \\
(\AA)\end{array}$ & $\begin{array}{c}p \\
(\%)\end{array}$ & $\theta$ & $p_{\text {corr }}$ & $\theta_{\text {corr }}$ \\
\hline $4125 \ldots$ & $2.70 \pm 0.44$ & $36.7 \pm 5.1$ & 2.82 & 33.12 \\
\hline $4375 \ldots$ & $2.60 \pm 0.16$ & $34.3 \pm 1.6$ & 2.76 & 30.60 \\
\hline $4625 \ldots \ldots$ & $3.11 \pm 0.09$ & $36.3 \pm 1.0$ & 3.25 & 33.00 \\
\hline $4875 \ldots \ldots$ & $2.75 \pm 0.09$ & $36.4 \pm 0.7$ & 2.89 & 32.68 \\
\hline $5125 \ldots \ldots$ & $3.12 \pm 0.05$ & $34.9 \pm 0.6$ & 3.28 & 31.63 \\
\hline $5375 \ldots \ldots \ldots$ & $2.90 \pm 0.07$ & $35.4 \pm 0.6$ & 3.05 & 31.81 \\
\hline 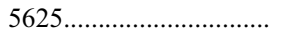 & $2.97 \pm 0.06$ & $35.0 \pm 0.4$ & 3.13 & 31.51 \\
\hline $5875 \ldots \ldots$ & $3.21 \pm 0.05$ & $32.7 \pm 0.5$ & 3.39 & 29.63 \\
\hline $6125 \ldots$. & $2.81 \pm 0.05$ & $35.5 \pm 0.4$ & 2.96 & 31.84 \\
\hline 6375. & $2.69 \pm 0.05$ & $35.4 \pm 0.4$ & 2.84 & 31.64 \\
\hline $6625 \ldots$ & $2.62 \pm 0.04$ & $34.7 \pm 0.5$ & 2.78 & 30.92 \\
\hline 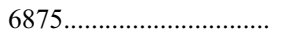 & $2.66 \pm 0.04$ & $34.5 \pm 0.6$ & 2.82 & 30.87 \\
\hline $7125 \ldots \ldots \ldots$ & $2.51 \pm 0.05$ & $35.3 \pm 0.5$ & 2.66 & 31.43 \\
\hline $7375 \ldots \ldots$ & $2.35 \pm 0.06$ & $35.8 \pm 0.8$ & 2.49 & 31.76 \\
\hline 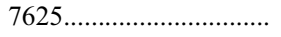 & $2.34 \pm 0.06$ & $34.8 \pm 0.9$ & 2.49 & 30.88 \\
\hline .................. & $2.27 \pm 0.08$ & $36.8 \pm 1.2$ & 2.39 & 32.75 \\
\hline 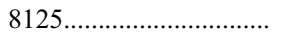 & $2.18 \pm 0.13$ & $33.1 \pm 1.4$ & 2.34 & 29.18 \\
\hline
\end{tabular}


TABLE 5

S233

\begin{tabular}{|c|c|c|c|c|}
\hline $\begin{array}{c}\lambda \text { bin } \\
(\AA)\end{array}$ & $\begin{array}{c}p \\
(\%)\end{array}$ & $\theta$ & $p_{\text {corr }}$ & $\theta_{\text {corr }}$ \\
\hline $4125 .$. & $1.82 \pm 0.42$ & $53.6 \pm 5.7$ & 1.74 & 47.76 \\
\hline $4375 \ldots \ldots \ldots$ & $2.03 \pm 0.18$ & $44.9 \pm 2.5$ & 2.06 & 39.59 \\
\hline $4625 \ldots \ldots \ldots$ & $1.84 \pm 0.12$ & $44.8 \pm 1.8$ & 1.88 & 38.82 \\
\hline $4875 \ldots$ & $1.90 \pm 0.11$ & $43.5 \pm 1.6$ & 1.96 & 37.67 \\
\hline $5125 \ldots$ & $1.92 \pm 0.08$ & $41.3 \pm 1.2$ & 2.01 & 35.64 \\
\hline $5375 \ldots \ldots$ & $1.94 \pm 0.07$ & $44.0 \pm 1.4$ & 1.99 & 38.20 \\
\hline $5625 \ldots \ldots$ & $1.74 \pm 0.10$ & $42.6 \pm 1.1$ & 1.82 & 36.21 \\
\hline $5875 \ldots \ldots$ & $1.78 \pm 0.07$ & $44.6 \pm 1.1$ & 1.83 & 38.25 \\
\hline 6125 & $1.85 \pm 0.05$ & $43.4 \pm 0.9$ & 1.91 & 37.48 \\
\hline $6375 \ldots$ & $1.84 \pm 0.06$ & $43.0 \pm 1.0$ & 1.91 & 37.11 \\
\hline $6625 \ldots \ldots$ & $1.74 \pm 0.06$ & $44.5 \pm 1.0$ & 1.79 & 38.25 \\
\hline $6875 \ldots \ldots$ & $1.63 \pm 0.07$ & $47.2 \pm 1.2$ & 1.64 & 40.57 \\
\hline $7125 \ldots$ & $1.62 \pm 0.06$ & $44.0 \pm 1.3$ & 1.67 & 37.53 \\
\hline  & $1.52 \pm 0.09$ & $45.5 \pm 1.4$ & 1.56 & 38.63 \\
\hline $7625 \ldots \ldots$ & $1.36 \pm 0.08$ & $52.3 \pm 1.8$ & 1.31 & 44.58 \\
\hline 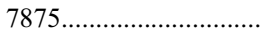 & $1.34 \pm 0.14$ & $37.6 \pm 2.4$ & 1.47 & 30.82 \\
\hline 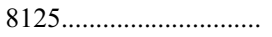 & $1.02 \pm 0.15$ & $51.6 \pm 3.8$ & 1.00 & 41.77 \\
\hline
\end{tabular}

low, at best. So, it would appear that there is something intrinsically different (and interesting) about the grains producing the observed polarization in M31 from those in our Galaxy. We intend to investigate this behavior in much greater depth after our sample of M31 globular clusters has been increased.

Previously, only S78 had enough wavelength coverage for a Serkowski fit. Our new data, which have better wavelength coverage and better $\mathrm{S} / \mathrm{N}$, show that $\lambda_{\max } \sim 4775 \AA$ for $\mathrm{S} 78$, very different from that found by Martin \& Shawl (1982). Their value of $3500 \AA$ is at the extreme low end of $\lambda_{\max }$ values measured in the Galaxy (e.g., Serkowski et al. 1975). The unusual sight line toward HD 210121, which has one of the lowest $R_{V}$ values ever measured, has $\lambda_{\max } \sim 3800 \AA$ (Larson, Whittet, \& Hough 1996). None of the $\sim 20$ sight lines measured in the LMC show $\lambda_{\max }$ values below $4900 \AA$ (Clayton et al. 1983). Only a few sight lines have been observed in the
SMC and the $\mathrm{S} / \mathrm{N}$ is relatively low, but $\lambda_{\max }$ lies in the normal Galactic range (Rodrigues et al. 1997).

The fits for the four sight lines in M31 reveal values of $\lambda_{\max }$ ranging from 4800 to $5500 \AA$. These are very average values of $\lambda_{\max }$ as measured in the Galaxy and the Magellanic Clouds. It has been found that $R_{V} \sim 5.5 \lambda_{\max }$ (Serkowski et al. 1975, Whittet \& van Breda 1978; Clayton \& Mathis 1988). The range of $\lambda_{\max }$ measured in M31 corresponds to $R_{V}$ values of 2.7-3.1. The range in $R_{V}$ seen in the Galaxy is $2.5-5.5$. As a result, at least for this very small sample, M31 does not show evidence for sight lines with greater than average grain size, if the nature of the grains is the same as for the Galaxy. This result is in contrast to the paucity of small grains inferred from IRAS colors (Xu \& Helou 1994).

The polarizing efficiency of the M31 dust grains can be estimated using the ratio of peak linear polarization to color excess, $p_{\max } / E(B-V)$. While the quantity is often equated with

TABLE 6

BAADE 327

\begin{tabular}{|c|c|c|c|c|}
\hline $\begin{array}{c}\lambda \text { bin } \\
(\AA)\end{array}$ & $\begin{array}{c}p \\
(\%)\end{array}$ & $\theta$ & $p_{\text {corr }}$ & $\theta_{\text {corr }}$ \\
\hline $4125 \ldots \ldots \ldots$ & $5.72 \pm 3.26$ & $74.0 \pm 9.7$ & 5.40 & 73.01 \\
\hline 4375 .............................. & $6.49 \pm 1.68$ & $45.8 \pm 5.2$ & 6.50 & 44.10 \\
\hline $4625 \ldots$ & $7.31 \pm 1.05$ & $54.5 \pm 3.3$ & 7.19 & 53.02 \\
\hline $4875 \ldots$ & $8.81 \pm 0.74$ & $58.3 \pm 1.9$ & 8.64 & 57.10 \\
\hline $5125 \ldots$ & $9.09 \pm 0.45$ & $53.0 \pm 1.3$ & 8.99 & 51.78 \\
\hline$\ldots$ & $9.37 \pm 0.31$ & $56.1 \pm 0.8$ & 9.21 & 55.86 \\
\hline $5625 \ldots \ldots \ldots \ldots \ldots \ldots$ & $9.57 \pm 0.23$ & $53.9 \pm 0.7$ & 9.46 & 52.73 \\
\hline $5875 \ldots \ldots \ldots$ & $9.18 \pm 0.20$ & $53.1 \pm 0.6$ & 9.08 & 51.85 \\
\hline $6125 \ldots \ldots \ldots$ & $9.04 \pm 0.18$ & $53.8 \pm 0.4$ & 8.93 & 52.58 \\
\hline $6375 \ldots \ldots \ldots$ & $8.63 \pm 0.14$ & $54.7 \pm 0.5$ & 8.51 & 53.42 \\
\hline $6625 \ldots \ldots \ldots \ldots \ldots \ldots \ldots$ & $8.62 \pm 0.15$ & $54.9 \pm 0.4$ & 8.50 & 53.63 \\
\hline  & $8.37 \pm 0.13$ & $55.2 \pm 0.4$ & 8.25 & 54.00 \\
\hline $7125 \ldots \ldots \ldots$ & $7.74 \pm 0.13$ & $54.9 \pm 0.5$ & 7.62 & 53.59 \\
\hline 7375 & $7.33 \pm 0.13$ & $53.4 \pm 0.5$ & 7.23 & 52.04 \\
\hline $7625 \ldots \ldots \ldots$ & $7.28 \pm 0.12$ & $54.4 \pm 0.5$ & 7.17 & 53.05 \\
\hline $7875 \ldots \ldots$ & $7.15 \pm 0.19$ & $54.0 \pm 0.5$ & 7.05 & 52.61 \\
\hline $8125 \ldots \ldots \ldots \ldots \ldots \ldots \ldots \ldots$ & $6.72 \pm 0.22$ & $55.1 \pm 0.8$ & 6.60 & 53.66 \\
\hline
\end{tabular}




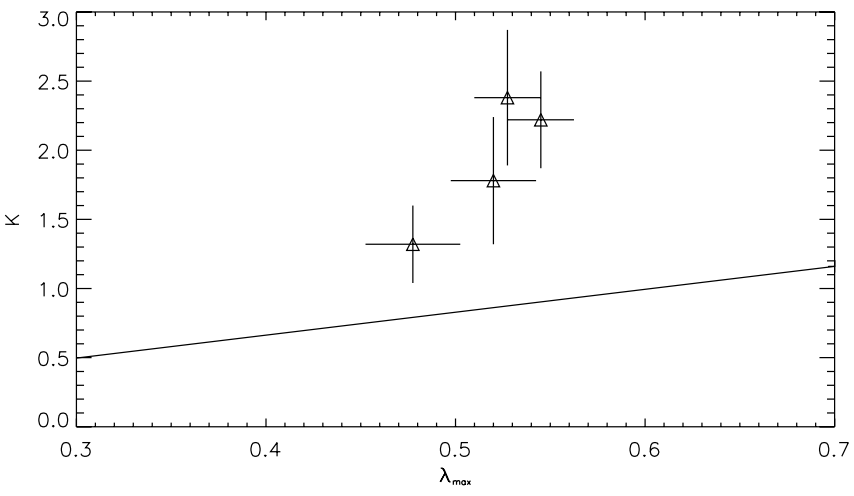

FIG. 2.-Serkowski fit parameters $K$ and $\lambda_{\max }$ plotted for the four M31 globular clusters. The error bars are $1 \sigma$. The line represents the $K=1.66 \lambda_{\max }$ relationship, which holds for Galactic interstellar polarization (Whittet et al. 1992).

the degree of grain alignment efficiency, it is also a function of the grain's nonspherical nature, as well as the angle between the alignment axis and the line of sight. However, for brevity, we simply refer to the value as the "efficiency ratio." The maximum value of this ratio found in the Galaxy is $9 \% \mathrm{mag}^{-1}$ (Serkowski et al. 1975; Clayton \& Cardelli 1988). We corrected the estimated reddenings for a Galactic foreground of $E(B-V)=$ $0.1 \mathrm{mag}$ (Barmby et al. 2000). The values of $p_{\max } / E(B-V)$ for our sample are given in Table 1 . They range from $7.1 \%$ to $15.3 \% \mathrm{mag}^{-1}$. These values generally support the results of Martin \& Shawl (1982) and imply a similar polarization efficiency in M31 and the Galaxy. The values of $p_{\max } / E(B-V)$ for our sample are all near the maximum value, but some bias is expected since sight lines known to have significant interstellar polarization were selected for inclusion in this sample. The polarization efficiency toward S233 seems very high, but the amount of reddening is fairly small, and the uncertainty is a considerable fraction of the $E(B-V)$ value itself.

Baade 327 is of interest since it is both the most luminous globular cluster known and also the most reddened globular cluster in M31 (Barmby, Perrett, \& Bridges 2002). Van den Bergh (1968) suggested that it is strange that the brightest globular cluster in M31 would also be the most reddened. This coincidence led to speculation that perhaps the value of $R_{V}$ might be small along this sight line resulting in less total extinction, $A_{V}$. Our result for Baade $327, \lambda_{\max } \sim 5450 \AA$ implies that the dust along this sight line has the Galactic average, $R_{V} \sim 3.1$. Even if $R_{V}=2.5$, as suggested by Kron \& Mayall (1960), Baade 327 only drops to the second most luminous globular cluster (Barmby et al. 2002). They also point out that the most reddened globular clusters lie close to the center of M31, and those close to the center of M31 seem to be brighter. The polarization efficiency toward Baade 327 is tantalizingly high, particularly given its large color excess and its anticipated favorable viewing geometry (i.e., the angle between alignment and line of sight; Martin \& Shawl 1979, 1982). Clearly, additional observations of M31 globular clusters would provide further insight into the dust environment in that galaxy.

We thank Pauline Barmby for providing us with reddening estimates for the globular clusters. We also thank Xiaohui Fan and Gary Schmidt for the use of the CCD Spectropolarimeter at the MMT during periods when weather conditions were unsuitable for the primary science program. This study has been supported by NASA ATP grant NAG5-9203.

\section{REFERENCES}

Barmby, P., Huchra, J. P., Brodie, J. P., Forbes, D. A., Schroder, L. L., \& Grillmair, C. J. 2000, AJ, 119, 727

Barmby, P., Perrett, K. M., \& Bridges, T. J. 2002, MNRAS, 329, 461

Bianchi, L., Clayton, G. C., Hutchings, J. B., Massey, P., \& Bohlin, R. C. 1996, ApJ, 471, 203

Cardelli, J. A., Clayton, G. C., \& Mathis, J. S. 1989, ApJ, 345, 245 (CCM)

Clayton, G. C., \& Cardelli, J. A. 1988, AJ, 96, 695

Clayton, G. C., Green, J., Wolff, M. J., Zellner, N. E. B., Code, A. D., \& Davidsen, A. F. 1996, ApJ, 460, 313

Clayton, G. C., Martin, P. G., \& Thompson, I. 1983, ApJ, 265, 194

Clayton, G. C., \& Mathis, J. S. 1988, ApJ, 327, 911

Clayton, G. C., Wolff, M. J., Allen, R. G., \& Lupie, O. L. 1995, ApJ, 445, 947

Freedman, W. L., \& Madore, B. F. 1990, ApJ, 365, 186

Gordon, K. D., \& Clayton, G. C. 1998, ApJ, 500, 816

Gordon, K. D., Clayton, G. C., Misselt, K. A., Landolt, A. U., \& Wolff, M. J. 2003, ApJ, 594, 279

Hiltner, W. A. 1958, ApJ, 128, 9

Iye, M., \& Richter, O.-G. 1985, A\&A, 144, 471

Kron, G. E., \& Mayall, N. U. 1960, AJ, 65, 581

Larson, K. A., Whittet, D. C. B., \& Hough, J. H. 1996, ApJ, 472, 755

Lequeux, J. 2000, in Proc. 232, The Interstellar Medium in M31 and M33, ed. E. M. Berkhuijsen, R. Beck, \& R. A. M. Walterbos (Aachen: Shaker), 63

Martin, P. G., Clayton, G. C., \& Wolff, M. J. 1999, ApJ, 510, 905

Martin, P. G., \& Shawl, S. J. 1979, ApJ, 231, L57 1982, ApJ, 253, 86

Massey, P., Armandroff, T. E., Pyke, R., Patel, K., \& Wilson, C. D. 1995, AJ, 110,2715

Massey, P., Strobel, K., Barnes, J. V., \& Anderson, E. 1988, ApJ, 328, 315
Misselt, K., Clayton, G. C., \& Gordon, K. D. 1999, ApJ, 515, 128

Nedialkov, P., \& Veltchev, T. 1999, Rev. Mexicana Astron. Astrofis., submitted (astro-ph/9911262)

Nordsieck, K. H., \& Harris, W. 1996, in ASP Conf. Ser. 97, Polarimetry of the Interstellar Medium, ed. W. G. Roberge \& D. C. B. Whittet (San Francisco: ASP), 100

Rodrigues, C. V., Magalhaes, A. M., Coyne, C. V., S. J., \& Piirola, V. 1997, ApJ, 485, 618

Sargent, W. L. W., Kowal, C. T., Hartwick, F. D. A., \& van den Bergh, S. 1977, AJ, 82, 947

Schmidt, G. D., Elston, R., \& Lupie, O. L. 1992, AJ, 104, 1563

Schmidt, G. D., Stockman, H. S., \& Smith, P. S. 1992, ApJ, 398, L57

Searle, L. 1983, Carnegie Inst. of Washington Yearbook, 82, 622

Serkowski, K., Mathewson, D. S., \& Ford, V. L. 1975, ApJ, 196, 261

Sharov, A. S., \& Lyutyj, V. M. 1989, AZh, 66, 462

van den Bergh, S. 1968, Observatory, 88, 168

Vetesnik, M. 1962, Bull. Astron. Inst. Czechoslovakia, 13, 180

Whittet, D. C. B., Martin, P. G., Hough, J. H., Rouse, M. F., Bailey, J. A., \& Axon, D. J. 1992, ApJ, 386, 562

Whittet, D. C. B., \& van Breda, I. G. 1978, A\&A, 66, 57

Wilking, B. A., Lebofsky, M. J., Martin, P. G., Rieke, G. H., \& Kemp, J. C. 1980, ApJ, 235, 905

Wilking, B. A., Lebofsky, M. J., \& Rieke, G. H. 1982, AJ, 87, 695

Wolff, M. J., Clayton, G. C., Martin, P. G., \& Schulte-Ladbeck, R. E. 1994, ApJ, 423, 412

Wolff, M. J., Nordsieck, K. H., \& Nook, M. A. 1996, AJ, 111, 856

Xu, C., \& Helou, G. 1994, ApJ, 426, 109 